\title{
Pseudo-slant submanifolds in cosymplectic space forms
}

\author{
Süleyman Dirik \\ Department of Statistic, \\ Faculty of Arts and Sciences, \\ Amasya University, Turkey \\ email: slymndirik@gmail.com
}

\author{
Mehmet Atçeken \\ Department of Mathematics, \\ Faculty of Arts and Sciences, \\ Gaziosmanpasa University, Turkey \\ email: mehmet.atceken382@gmail.com
}

\begin{abstract}
In this paper, we study the geometry of the pseudo-slant submanifolds of a cosymplectic space form. Necessary and sufficient conditions are given for a submanifold to be a pseudo-slant submanifold, pseudo-slant product, mixed geodesic and totally geodesic in cosymplectic manifolds. Finally, we give some results for totally umbilical pseudoslant submanifold in a cosymplectic manifold and cosymplectic space form.
\end{abstract}

\section{Introduction}

The differential geometry of slant submanifolds has shown an increasing development since B. Y. Chen [3, 4] defined slant submanifolds in complex manifolds as a natural generalization of both the invariant and anti-invariant submanifolds. Many research articles have been appeared on the existence of these submanifolds in different knows spaces. The slant submanifols of an almost contact metric manifolds were defined and studied by A. Lotta [2]. After, this type submanifolds were studied by J.L Cabrerizo et. al [7] of Sasakian manifolds. Recently, in [8] M. Atçeken studied slant and pseudo-slant submanifold

2010 Mathematics Subject Classification: 53C15, 53C25, 53C17, 53D15, 53D10 Key words and phrases: cosymplectic manifold, cosymplectic space form, pseudo-slant submanifold 
in (LCS $)_{\mathfrak{n}}$-manifolds. The notion of semi-slant submanifolds of an almost Hermitian manifold was introduced by N. Papagiuc [12]. Recently, A. Carriazo [1] defined and studied bi-slant immersions in almost Hermitian manifolds and simultaneously gave the notion of pseudo-slant submanifolds in almost Hermitian manifolds. The contact version of pseudo-slant submanifolds has been defined and studied by V. A. Khan and M. A. Khan [15].

In this paper, we study pseudo-slant submanifolds of a cosymplectic manifold. In section 2, we review basic formulas and definitions for a cosymplectic manifold and their submanifolds. In section 3 , we recall the definition and some basic results of a pseudo-slant submanifold of an almost contact metric manifold. In section 4, we give some new results for totally umbilical pseudoslant submanifold in a cosymplectic manifold $\widetilde{M}$ and cosymplectic space form $\widetilde{M}(c)$.

\section{Preliminaries}

In this section, we give some notations used throughout this paper. We recall some necessary fact and formulas from the theory of Cosymplectic manifolds and their submanifols.

Let $\widetilde{M}$ be a $(2 m+1)$-dimensional almost contact metric manifold together with a metric tensor $\mathrm{g}$, a tensor field $\phi$ of type $(1,1)$, a vector field $\xi$ and a 1-form $\eta$ on $\widetilde{M}$ which satisfy

$$
\begin{aligned}
& \phi^{2} X=-X+\eta(X) \xi, \\
& \phi \xi=0, \eta(\phi X)=0, \eta(\xi)=1, \eta(X)=g(X, \xi)
\end{aligned}
$$

and

$$
g(\phi X, \phi Y)=g(X, Y)-\eta(X) \eta(Y), \quad g(\phi X, Y)=-g(X, \phi Y)
$$

for any vector fields $X, Y$ on $\widetilde{M}$. An almost contact structure $(\phi, \xi, \eta)$ is said to be normal if the almost complex structure $J$ on the product manifold $\widetilde{M} \times R$ given by

$$
J\left(X, f \frac{d}{d t}\right)=\left(\phi X-f \xi, \eta(X) \frac{d}{d t}\right)
$$

where $f$ is the $C^{\infty}$ - function on $\widetilde{M} \times R$. The condition for normality in terms of $\phi, \xi$ and $\eta$ is $[\phi, \phi]+2 d \eta \otimes \xi=0$ on $\widetilde{M}$, where $[\phi, \phi](X, Y)=\phi^{2}$ $[X, Y]+[\phi X, \phi Y]-\phi[\phi X, Y]-\phi[X, \phi Y]$ is the Nijenhuis tensor of $\phi$. Finally the fundamental $2-$ form $\Phi$ is defined by $\Phi(X, Y)=g(X, \phi Y)$. 
An almost contact metric structure $(\phi, \xi, \eta, g)$ is said to be cosymplectic, if it is normal and both $\Phi$ and are $\eta$ closed. So we have on a cosymplectic manifold $\widetilde{M}$

$$
\left(\widetilde{\nabla}_{X} \phi\right) Y=0
$$

for any vector fields $X, Y$ on $\widetilde{M}$. (4) implies that

$$
\widetilde{\nabla}_{X} \xi=0
$$

for any $\mathrm{X} \in \Gamma(\mathrm{TM})$, that is $\xi$ is a killing vector field.

Let $\widetilde{R}$ be the curvature tensor of the connection $\widetilde{\nabla}$. The sectional curvature of a $\phi$ - sectional is called a $\phi$ - sectional curvature. A cosymplectic manifold with constant $\phi$ - sectional curvature $\mathrm{c}$ is said to be a cosymplectic space form and it is denoted by $\widetilde{M}(c)$. The curvature tensor $\widetilde{R}$ of a cosymplectic space form $\widetilde{M}(c)$ is given by

$$
\begin{aligned}
\widetilde{R}(X, Y) Z= & \frac{c}{4}\{g(Y, Z) X-g(X, Z) Y+\eta(X) \eta(Z) Y-\eta(Y) \eta(Z) X \\
& +\eta(Y) g(X, Z) \xi-\eta(X) g(Y, Z) \xi+g(\phi Y, Z) \phi X \\
& +g(X, \phi Z) \phi Y+2 g(X, \phi Y) \phi Z\}
\end{aligned}
$$

for any vector fields $X, Y, Z$ tangent to $\widetilde{M}[13]$.

Now, let $M$ be an isometrical immersed submanifold of a contact metric manifold $\widetilde{M}$ and denote by the same symbol $g$ the Riemanian metric induced on $M$. Let $\Gamma(\mathrm{TM})$ and $\Gamma\left(\mathrm{T}^{\perp} \mathrm{M}\right)$ be the diferential vector fields set tangent and normal to $M$, respectively. Also we denote by $\nabla$ and $\nabla^{\perp}$ induced connections on $\Gamma(\mathrm{TM})$ and $\Gamma\left(\mathrm{T}^{\perp} \mathrm{M}\right)$, respectively. Then the Gauss and Weingarten formulas are, respectively, given by

$$
\widetilde{\nabla}_{X} Y=\nabla_{X} Y+h(X, Y)
$$

and

$$
\widetilde{\nabla}_{X} V=-A_{V} X+\nabla_{X}^{\perp} V
$$

for all $X, Y \in \Gamma(T M)$ and $V \in \Gamma\left(T^{\perp} M\right)$, where $h$ and $A_{V}$ are the second fundamental form and shape operator for the immersed of $M$ into $\widetilde{M}$, respectively. They are related as

$$
g\left(A_{V} X, Y\right)=g(h(X, Y), V)
$$


We put

$$
h_{i j}^{r}=g\left(h\left(e_{i}, e_{j}\right), e_{r}\right) \text { and }\|h\|^{2}=\sum_{i, j=1}^{n} g\left(h\left(e_{i}, e_{j}\right), h\left(e_{i}, e_{j}\right)\right) \text {, }
$$

where, $\left\{e_{1}, e_{2}, \ldots, e_{n}\right\}$ is an orthonormal basis of $\Gamma(T M)$ and $\left\{e_{n+1}, \ldots, e_{2 m+1}\right\}$ is also orthonormal basis of $\Gamma\left(\mathrm{T}^{\perp} \mathrm{M}\right)$.

The mean curvature vector $\mathrm{H}$ of $M$ is given by

$$
H=\frac{1}{n} \operatorname{trace}(h)=\frac{1}{n} \sum_{i=1}^{n} h\left(e_{i}, e_{i}\right)
$$

A submanifold $M$ of an contact metric manifold $\widetilde{M}$ is said to be totally umbilical if

$$
h(X, Y)=g(X, Y) H
$$

where $\mathrm{H}$ is the mean curvature vector. A submanifold $M$ is said to be totally geodesic submanifold if $h(X, Y)=0$, for each $X, Y \in \Gamma(T M)$ and $M$ is said to be minimal submanifold if $\mathrm{H}=0$.

For any submanifold $\mathrm{M}$ of a Riemannian manifold $\widetilde{M}$, the equation of Gauss is given by

$$
\widetilde{R}(X, Y) Z=R(X, Y) Z+A_{h(X, Z)} Y-A_{h(Y, Z)} X+\left(\nabla_{X} h\right)(Y, Z)-\left(\nabla_{Y} h\right)(X, Z),
$$

where $\widetilde{R}$ and $R$ denote the Riemannian curvature tensor of $\widetilde{M}$ and $M$, respectively, where the covariant derivative $\nabla h$ of $h$ is defined by

$$
\left(\nabla_{X} h\right)(Y, Z)=\nabla_{X}^{\perp} h(Y, Z)-h\left(\nabla_{X} Y, Z\right)-h\left(\nabla_{X} Z, Y\right)
$$

for any $X, Y, Z \in \Gamma(T M)$.

The normal component of (12) is said to be the Codazzi equation is given by

$$
(\widetilde{R}(X, Y) Z)^{\perp}=\left(\nabla_{X} h\right)(Y, Z)-\left(\nabla_{Y} h\right)(X, Z),
$$

where $(\widetilde{R}(X, Y) Z)^{\perp}$ denotes the normal part of $\widetilde{R}(X, Y) Z$. If $(\widetilde{R}(X, Y) Z)^{\perp}=0$, then $M$ is said to be curvature-invariant submanifold of $\widetilde{M}$. The Ricci equation is given by

$$
g(\widetilde{R}(X, Y) V, U)=g\left(\widetilde{R}^{\perp}(X, Y) V, U\right)+g\left(\left[A_{U}, A_{V}\right] X, Y\right)
$$


for any $X, Y, \in \Gamma(T M)$ and $V, U \in \Gamma\left(T^{\perp} M\right)$, where $\widetilde{R}^{\perp}$ denotes the Riemannian curvature tensor of the normal $T^{\perp} M$ and if $\widetilde{R}^{\perp}=0$, then the normal connection of $M$ is called flat.

A cosymplectic manifold $\widetilde{M}$ is said to be $\eta$-Einstein if its Ricci tensor $S$ of type $(0,2)$ is of the from

$$
S(X, Y)=a g(X, Y)+b \eta(X) \eta(Y)
$$

where $a, b$ are smooth functions on $\widetilde{M}$. If $b=0$, then the manifold is called Einstein.

\section{Pseudo-slant submanifolds of a cosymplectic manifold}

In this section, we study pseudo-slant submanifolds in a cosymplectic manifold and we give some characterization results.

Let $M$ be a submanifold of an almost contact metric manifold $\widetilde{M}$. Then for any $\mathrm{X} \in \Gamma(\mathrm{TM})$, we can set

$$
\phi X=\mathrm{TX}+\mathrm{NX}
$$

where TX and NX denote the tangential and the normal components of $\phi X$, respectively. In the same way, for any $V \in \Gamma\left(T^{\perp} M\right)$, we can write

$$
\phi \mathrm{V}=\mathrm{tV}+\mathrm{nV},
$$

where $\mathrm{tV}$ (resp.nV) are tangential(resp. normal) components of $\phi \mathrm{V}$.

A submanifold $M$ is said to be invariant if $N$ is identically zero, that is, $\phi X \in \Gamma(T M)$ for all $X \in \Gamma(T M)$. On the other hand, $M$ is said to be antiinvariant if $T$ is identically zero, that is, $\phi X \in \Gamma\left(T^{\perp} M\right)$ for all $X \in \Gamma(T M)$.

Thus by using (1), (17) and (18), we obtain

$$
\mathrm{T}^{2}=-\mathrm{I}-\mathrm{tN}+\eta \otimes \xi, \quad \mathrm{NT}+\mathrm{nN}=0
$$

and

$$
\mathrm{n}^{2}=-\mathrm{I}-\mathrm{Nt}, \quad \mathrm{Tt}+\mathrm{tn}=0 .
$$

Furthermore, the covariant derivatives of the tensor field $T, N, t$ and $n$ are, respectively, defined by

$$
\begin{aligned}
\left(\nabla_{X} \mathrm{~T}\right) \mathrm{Y} & =\nabla_{\mathrm{X}} \mathrm{TY}-\mathrm{T} \nabla_{\mathrm{X}} \mathrm{Y} \\
\left(\nabla_{\mathrm{X}} \mathrm{N}\right) \mathrm{Y} & =\nabla_{\mathrm{X}}^{\perp} \mathrm{NY}-\mathrm{N} \nabla_{\mathrm{X}} \mathrm{Y} \\
\left(\nabla_{\mathrm{X}} \mathrm{t}\right) \mathrm{V} & =\nabla_{\mathrm{X}} \mathrm{t} \mathrm{V}-\mathrm{t} \nabla_{\mathrm{X}}^{\perp} \mathrm{V}
\end{aligned}
$$


and

$$
\left(\nabla_{X} n\right) V=\nabla_{X}^{\frac{1}{X}} n V-n \nabla_{X}^{\perp} V
$$

Furthermore, for any $X, Y \in \Gamma(T M)$, we have $g(T X, Y)=-g(X, T Y)$ and $V, U \in$ $\Gamma\left(T^{\perp} M\right)$. By using (3), (17) and (18), we have $g(U, n V)=-g(n U, V)$. These show that $T$ and $n$ are also skew-symmetric tensor fields. Moreover, for any $\mathrm{X} \in \Gamma(\mathrm{TM})$ and $\mathrm{V} \in \Gamma\left(\mathrm{T}^{\perp} \mathrm{M}\right)$, we have

$$
g(N X, V)=-g(X, t V),
$$

which gives the relation between $\mathrm{N}$ and $\mathrm{t}$.

Taking into account (6) and (15), we have

$$
\begin{aligned}
g\left(\widetilde{R}^{\perp}(X, Y) V, U\right)= & \frac{c}{4}\{g(X, t V) g(U, N Y)-g(Y, t V) g(N X, U) \\
& +2 g(X, T Y) g(n V, U)\}+g\left(\left[A_{V}, A_{U}\right] X, Y\right)
\end{aligned}
$$

for any $\mathrm{X}, \mathrm{Y} \in \Gamma(\mathrm{TM})$ and $\mathrm{V}, \mathrm{U} \in \Gamma\left(\mathrm{T}^{\perp} \mathrm{M}\right)$.

By using (6) and (12), the Riemanian curvature tensor $\mathrm{R}$ of an immersed submanifold $M$ of a cosymplectic space form $\widetilde{M}(c)$ is given by

$$
\begin{aligned}
R(X, Y) Z= & \frac{c}{4}\{g(Y, Z) X-g(X, Z) Y+\eta(X) \eta(Z) Y-\eta(Y) \eta(Z) X \\
& +\eta(Y) g(X, Z) \xi-\eta(X) g(Y, Z) \xi+g(X, \phi Z) \phi Y \\
& +g(\phi Y, Z) \phi X+2 g(X, \phi Y) \phi Z\}+A_{h(Y, Z)} X-A_{h(X, Z)} Y \\
& +\left(\nabla_{Y} h\right)(X, Z)-\left(\nabla_{X} h\right)(Y, Z) .
\end{aligned}
$$

Comparing the tangential and normal parts of the both sides of this equation, we have, following equations of Gauss and Codazzi equation respectively:

$$
\begin{aligned}
(R(X, Y) Z)^{\top}= & \frac{c}{4}\{g(Y, Z) X-g(X, Z) Y+\eta(X) \eta(Z) Y-\eta(Y) \eta(Z) X \\
& +\eta(Y) g(X, Z) \xi-\eta(X) g(Y, Z) \xi+g(X, T Z) T Y \\
& +g(T Y, Z) T X+2 g(X, T Y) T Z\}+A_{h(Y, Z)} X-A_{h(X, Z)} Y
\end{aligned}
$$

and

$$
\begin{aligned}
\left(\nabla_{X} h\right)(Y, Z)-\left(\nabla_{Y} h\right)(X, Z)= & \frac{c}{4}\{g(X, T Z) N Y+g(T Y, Z) N X \\
& +2 g(X, T Y) N Z\} .
\end{aligned}
$$

By an easy computation, we obtain the following formulas

$$
\left(\nabla_{X} T\right) Y=A_{N Y} X+\operatorname{th}(X, Y)
$$


and

$$
\left(\nabla_{X} N\right) Y=n h(X, Y)-h(X, T Y) .
$$

Similarly, for any $\mathrm{V} \in \Gamma\left(\mathrm{T}^{\perp} \mathrm{M}\right)$ and $\mathrm{X} \in \Gamma(\mathrm{TM})$, we obtain

$$
\left(\nabla_{X} \mathrm{t}\right) \mathrm{V}=\mathrm{A}_{\mathrm{nV}} \mathrm{X}-\mathrm{TA}_{\mathrm{V}} \mathrm{X}
$$

and

$$
\left(\nabla_{X} \mathrm{n}\right) \mathrm{V}=-\mathrm{h}(\mathrm{tV}, \mathrm{X})-\mathrm{NA} \mathrm{V} .
$$

Since $M$ is tangent to $\xi$, making use of (5), (7), (9) and (17), we obtain

$$
\nabla_{X} \xi=0, \quad h(X, \xi)=0, \quad A_{V} \xi=0
$$

for all $\mathrm{V} \in \Gamma\left(\mathrm{T}^{\perp} \mathrm{M}\right)$ and $\mathrm{X} \in \Gamma(\mathrm{TM})$.

Definition 1 A submanifold $M$ of an almost contact metric manifold $\widetilde{M}$ is said to be slant submanifold if for any $x \in M$ and $X \in \mathrm{T}_{\chi} \mathrm{M}-\xi$ the angle between $\mathrm{T}_{\chi} \mathrm{M}$ and $\phi \mathrm{X}$ is constant. The constant angle $\left[0, \frac{\pi}{2}\right]$ is then called slant angle of $\mathrm{M}$. If $\theta=0$, the submanifold is invariant submanifold, if $\theta=\frac{\pi}{2}$ then, it is anti-invariant submanifold, if $\theta \in\left(0, \frac{\pi}{2}\right)$ then it is proper slant submanifold [2].

In almost contact metric manifolds, J. L Cabrerizo [7] proved the following theorem.

Theorem 1 Let $M$ be a slant submanifold of an almost contact metric manifold $\widetilde{M}$ such that $\xi \in \Gamma(\mathrm{TM})$. Then, $M$ is slant submanifold if and only if there exists a constant $\lambda \in[0,1]$ such that

$$
\mathrm{T}^{2}=-\lambda(\mathrm{I}-\eta \otimes \xi)
$$

furthermore, in this case, if $\theta$ is the slant angle of $\mathrm{M}$, then $\lambda=\cos ^{2} \theta[7]$.

Corollary 1 Let $\mathrm{M}$ be a slant submanifold of an almost contact metric manifold $\widetilde{M}$ with slant angle $\theta$. Then for any $\mathrm{X}, \mathrm{Y} \in \Gamma(\mathrm{TM})$, we have

$$
g(T X, T Y)=\cos ^{2} \theta\{g(X, Y)-\eta(X) \eta(Y)\}
$$

and

$$
g(N X, N Y)=\sin ^{2} \theta\{g(X, Y)-\eta(X) \eta(Y)\}[\eta] .
$$


Let $M$ be a slant submanifold of an almost contact metric manifold $\widetilde{M}$ with slant angle $\theta$. Then for any $X \in \Gamma(T M)$, from (19) and (35), we have

$$
-\cos ^{2} \theta(X-\eta(X) \xi)=-X+\eta(X) \xi-t N X
$$

from which

$$
\mathrm{tNX}=-\sin ^{2} \theta(X-\eta(X) \xi)
$$

by using (37),

$$
N^{2} X=-\sin ^{2} \theta(X-\eta(X) \xi)
$$

from (38) and (39) we, obtain

$$
\mathrm{N}^{2}=\mathrm{tN}
$$

It is well known that th $=0$ plays an important role in the geometry of submanifolds. This means that the induced structure $T$ is a cosymplectic structure on $M$.

By using (30) and (34), we obtain

$$
\eta\left(\left(\nabla_{X} \top\right) Y\right)=0
$$

for $X, Y \in \Gamma\left(D_{\theta}\right)$.

Definition 2 We say that $M$ is a pseudo-slant submanifold of an almost contact metric manifold $\widetilde{M}$ if there exist two orthogonal distributions $\mathrm{D}_{\theta}$ and $\mathrm{D}^{\perp}$ on $\mathrm{M}$ such that

(a) $\mathrm{TM}$ admits the orthogonal direct decomposition $\mathrm{TM}=\mathrm{D}^{\perp} \oplus \mathrm{D}_{\theta}, \xi \in$ $\Gamma\left(D_{\theta}\right)$,

(b) The distribution $\mathrm{D}^{\perp}$ is anti-invariant(totally-real) i.e., $\phi \mathrm{D}^{\perp} \subset\left(\mathrm{T}^{\perp} \mathrm{M}\right)$,

(c) The distribution $\mathrm{D}_{\theta}$ is a slant, that is, the slant between of $\mathrm{D}_{\theta}$ and $\phi\left(\mathrm{D}_{\theta}\right)$ is a constant [15].

Let $d_{1}=\operatorname{dim}\left(D^{\perp}\right)$ and $d_{2}=\operatorname{dim}\left(D_{\theta}\right)$. We distinguish the following five cases.

(i) If $d_{2}=0$ or $\theta=\frac{\pi}{2}$, then $M$ is an anti-invariant submanifold.

(ii) If $d_{1}=0$ and $\theta=0$, then $M$ is invariant submanifold.

(iii) If $d_{1}=0$ and $\theta \neq\left\{0, \frac{\pi}{2}\right\}$, then $M$ is a proper slant submanifold.

(iv) If $d_{2} d_{1} \neq 0$ and $\theta=0$, then $M$ is a semi-invariant submanifold.

(v) If $d_{2} d_{1} \neq 0$ and $\theta \neq\left\{0, \frac{\pi}{2}\right\}$, then $M$ is a proper pseudo-slant submanifold. 
By $\mu$ we denote the orthogonal complementary of $\phi(T M)$ in $T^{\perp} M$, then we have the following sum

$$
\mathrm{T}^{\perp} \mathrm{M}=\mathrm{N}\left(\mathrm{D}^{\perp}\right) \oplus \mathrm{N}\left(\mathrm{D}_{\theta}\right) \oplus \mu .
$$

Let $M$ be a proper pseudo-slant submanifold of a cosymplectic manifold $\widetilde{M}$. Then for any $Z, W \in \Gamma\left(D^{\perp}\right)$ and $U \in \Gamma(T M)$, also by using (4), (7) and (9), we have

$$
\begin{aligned}
& g\left(A_{N Z} W-A_{N W Z} Z, U\right)=g(h(W, U), N Z)-g(h(Z, U), N W) \\
& =\mathrm{g}(\widetilde{\nabla} \mathrm{u} W, \phi \mathrm{Z})-\mathrm{g}(\widetilde{\nabla} \mathrm{u} Z, \phi \mathrm{W}) \\
& =\mathrm{g}\left(\phi \widetilde{\nabla}_{\mathrm{U}} \mathrm{Z}, \mathrm{W}\right)-\mathrm{g}(\phi \widetilde{\nabla} \mathrm{u} \mathrm{W}, \mathrm{Z}) \\
& =g(\widetilde{\nabla} u \phi Z-(\widetilde{\nabla} u \phi) Z, W)+g\left(\left(\widetilde{\nabla}_{u} \phi\right) W-\widetilde{\nabla}_{u} \phi W, Z\right) \\
& =g(\widetilde{\nabla} \mathrm{u} \phi \mathrm{Z}, \mathrm{W})-\mathrm{g}\left(\widetilde{\nabla}_{\mathrm{u}} \phi \mathrm{W}, \mathrm{Z}\right) \\
& =-g\left(A_{N Z} \mathrm{U}, W\right)+g\left(A_{N W} \mathrm{U}, \mathrm{Z}\right) \\
& =g\left(A_{N W} Z-A_{N Z} W, U\right) \text {. }
\end{aligned}
$$

It follows that

$$
A_{N Z} W=A_{N W} Z
$$

Theorem 2 Let $M$ be a proper pseudo-slant submanifold of a cosymplectic manifold $\widetilde{M}$. Then the tensor $\mathrm{N}$ is parallel if and only if the tensor $\mathrm{t}$ is parallel.

Proof. By using (9), (31) and (32), we have

$$
\begin{aligned}
g\left(\left(\nabla_{X} N\right) Y, V\right) & =g(n h(X, Y), V)-g(h(X, T Y), V) \\
& =-g(h(X, Y), n V)-g\left(A_{V} X, T Y\right) \\
& =-g\left(A_{n V} X, Y\right)+g\left(T A_{V} X, Y\right) \\
& =g\left(-A_{n V} X+T A_{V} X, Y\right)=g\left(\left(\nabla_{X} t\right) V, Y\right),
\end{aligned}
$$

for any $X, Y \in \Gamma(T M)$ and $V \in \Gamma\left(T^{\perp} M\right)$. This proves our assertion.

Theorem 3 Let $M$ be a proper pseudo-slant submanifold of a cosymplectic manifold $\widetilde{M}$. Then the tensor $\mathrm{N}$ is parallel if and only if

$$
A_{V} T Y=-A_{n V} Y
$$

for any $\mathrm{Y} \in \Gamma(\mathrm{TM})$ and $\mathrm{V} \in \Gamma\left(\mathrm{T}^{\perp} \mathrm{M}\right)$. 
Proof. By using (9) and (31), we have

$$
\begin{aligned}
g\left(\left(\nabla_{X} N\right) Y, V\right) & =g(n h(X, Y), V)-g(h(X, T Y), V) \\
& =-g(h(X, Y), n V)-g\left(A_{V} T Y, X\right) \\
& =-g\left(A_{n V} X, Y\right)-g\left(A_{V} T Y, X\right)
\end{aligned}
$$

for any $X, Y \in \Gamma(T M)$ and $V \in \Gamma\left(T^{\perp} M\right)$. This proves our assertion.

Theorem 4 Let $M$ be a proper pseudo-slant submanifold of a cosymplectic manifold $\widetilde{M}$. The covariant derivation of $\mathrm{T}$ is skew-symmetric, that is

$$
g\left(\left(\nabla_{X} \mathrm{~T}\right) \mathrm{Y}, \mathrm{Z}\right)=-\mathrm{g}\left(\left(\nabla_{X} \mathrm{~T}\right) \mathrm{Z}, \mathrm{Y}\right),
$$

for any $\mathrm{X}, \mathrm{Y}, \mathrm{Z} \in \Gamma(\mathrm{TM})$.

Proof. For any $X, Y, Z \in \Gamma(T M)$, by using (9), (25) and (30), we obtain

$$
\begin{aligned}
g\left(\left(\nabla_{X} T\right) Y, Z\right) & =g\left(A_{N Y} X+\operatorname{th}(X, Y), Z\right) \\
& =g(h(X, Z), N Y)-g(h(X, Y), N Z) \\
& =-g(\operatorname{th}(X, Z), Y)-g\left(A_{N Z} X, Y\right) \\
& =-g\left(A_{N Z} X+\operatorname{th}(X, Z), Y\right) \\
& =-g\left(\left(\nabla_{X} T\right) Z, Y\right) .
\end{aligned}
$$

This complete the proof.

Theorem 5 Let $\mathrm{M}$ be a proper pseudo-slant submanifold of a cosymplectic manifold $\widetilde{M}$. Then the tensor $\mathrm{T}$ is parallel if and only if

$$
A_{N Y} X=A_{N X} Y
$$

for any $\mathrm{X}, \mathrm{Y} \in \Gamma(\mathrm{TM})$.

Proof. For any $X, Y, Z \in \Gamma(T M)$, by using (9), (25) and (30), we obtain

$$
\begin{aligned}
g\left(\left(\nabla_{X} T\right) Y, Z\right) & =g\left(A_{N Y} X+\operatorname{th}(X, Y), Z\right) \\
& =g(h(X, Z), N Y)-g(h(X, Y), N Z) \\
& =g\left(A_{N Y} Z, X\right)-g\left(A_{N Z} Y, X\right)
\end{aligned}
$$

This complete the proof. 
Theorem 6 Let $M$ be a proper pseudo-slant submanifold of a cosymplectic manifold $\widetilde{M}$. The covariant derivation of $\mathrm{n}$ is skew-symmetric, that is,

$$
g\left(\left(\nabla_{X} n\right) V, U\right)=-g\left(\left(\nabla_{X} n\right) U, V\right)
$$

for any $\mathrm{X} \in \Gamma(\mathrm{TM})$ and $\mathrm{V}, \mathrm{U} \in \Gamma\left(\mathrm{T}^{\perp} \mathrm{M}\right)$.

Proof. For any $X \in \Gamma(T M)$ and $V, U \in \Gamma\left(T^{\perp} M\right)$, from (9), (25) and (33), we reach

$$
\begin{aligned}
g\left(\left(\nabla_{X} \mathrm{n}\right) \mathrm{V}, \mathrm{U}\right) & =g\left(-\mathrm{h}(\mathrm{tV}, X)-\mathrm{N} A_{V} X, \mathrm{U}\right) \\
& =g\left(-A_{\mathrm{U}} X, \mathrm{tV}\right)+\mathrm{g}\left(A_{V} X, \mathrm{tU}\right) \\
& =g\left(N A_{\mathrm{U}} X, \mathrm{~V}\right)+\mathrm{g}(\mathrm{h}(X, \mathrm{tU}), \mathrm{V}) \\
& =-g\left(-N A_{U} X-\mathrm{h}(X, \mathrm{tU}), \mathrm{V}\right) \\
& =-g\left(\left(\nabla_{X} \mathrm{n}\right) \mathrm{U}, \mathrm{V}\right) .
\end{aligned}
$$

This proves our assertion.

Theorem 7 Let $M$ be a proper pseudo-slant submanifold of a cosymplectic manifold $\widetilde{M}$. Then the tensor $n$ is parallel if and only if the shape operator $A_{V}$ of $M$ satisfies the condition

$$
A_{\mathrm{V}} \mathrm{tU}=\mathrm{A}_{\mathrm{u}} \mathrm{tV}
$$

for all $\mathrm{U}, \mathrm{V} \in \Gamma\left(\mathrm{T}^{\perp} \mathrm{M}\right)$.

Proof. From (9), (25) and (33), we have

$$
\begin{aligned}
g\left(\left(\nabla_{X} \mathrm{n}\right) \mathrm{V}, \mathrm{U}\right) & =-\mathrm{g}(\mathrm{h}(\mathrm{tV}, X), \mathrm{U})-\mathrm{g}\left(\mathrm{N} A_{V} X, \mathrm{U}\right) \\
& =-\mathrm{g}\left(A_{\mathrm{U}} \mathrm{tV}, \mathrm{X}\right)+\mathrm{g}\left(A_{V} X, \mathrm{tU}\right) \\
& =g\left(A_{V} \mathrm{tU}-A_{\mathrm{U}} \mathrm{tV}, X\right)
\end{aligned}
$$

for all $X \in \Gamma(T M)$. The proof is complete.

Theorem 8 Let $M$ be a proper pseudo-slant submanifold of a cosymplectic manifold $\widetilde{M}$. If tensor $\mathrm{n}$ is parallel then, $\mathrm{M}$ is totally geodesic submanifold of $\widetilde{M}$. 
Proof. Since $\boldsymbol{n}$ is parallel, from (33) and (17), we have

$$
h(t V, X)+\phi A_{V} X=0
$$

for all $\mathrm{X} \in \Gamma(\mathrm{TM})$ and $\mathrm{V} \in \Gamma\left(\mathrm{T}^{\perp} \mathrm{M}\right)$. Applying $\phi$ to (41) and taking into account (1) and (34), we obtain

$$
\begin{aligned}
0 & =\phi^{2} A_{V} X+\phi h(t V, X) \\
& =-A_{V} X+\eta\left(A_{V} X\right) \xi+\operatorname{th}(t V, X)+n h(t V, X) .
\end{aligned}
$$

This yields to

$$
-A_{V} X+\operatorname{th}(t V, X)=0 .
$$

On the other hand, also by using (9), (19), (25) and (40), we conclude that

$$
\begin{aligned}
g\left(A_{V} X, Z\right) & =g(\operatorname{th}(t \vee, X), Z)=-g(h(t V, X), N Z) \\
& =-g\left(A_{N Z} t V, X\right)=-g\left(A_{V} t N Z, X\right),
\end{aligned}
$$

for $Z \in \Gamma(T M)$. Taking into account of $t N Z=-Z+\eta(Z) \xi-T^{2} Z$, we obtain

$$
\begin{aligned}
g\left(A_{V} Z, X\right) & =-g\left(-A_{V} Z+\eta(Z) A_{V} \xi-A_{V} T^{2} Z, X\right) \\
& =g\left(A_{V} Z, X\right)+g\left(A_{V} X, T^{2} Z\right)
\end{aligned}
$$

that is,

$$
g\left(T^{2} A_{V} X, Z\right)=0 .
$$

Here, by using (36), we conclude

$$
0=-g\left(T A_{V} X, T Z\right)=-\cos ^{2} \theta g\left(A_{V} X, Z\right)
$$

for all $Z \in \Gamma(T M)$. Since $M$ is a proper pseudo-slant submanifold, we arrive at $A_{V}=0$, that is, $M$ is totally geodesic in $\widetilde{M}$.

Definition 3 A pseudo-slant submanifold $M$ of cosymplectic manifold $\widetilde{M}$ is said to be $\mathrm{D}_{\theta}$-geodesic (resp. $\mathrm{D}^{\perp}$-geodesic) if $\mathrm{h}(\mathrm{X}, \mathrm{Y})=0$ for $\mathrm{X}, \mathrm{Y} \in \Gamma\left(\mathrm{D}_{\theta}\right)$ (resp. $\mathrm{h}(\mathrm{Z}, \mathrm{W})=0$ for $\mathrm{Z}, \mathrm{W} \in \Gamma\left(\mathrm{D}^{\perp}\right)$ ). If $\mathrm{h}(\mathrm{X}, \mathrm{Z})=0, \mathrm{M}$ is called mixed geodesic submanifold, for any $\mathrm{X} \in \Gamma\left(\mathrm{D}_{\theta}\right)$ and $\mathrm{Z} \in \Gamma\left(\mathrm{D}^{\perp}\right)$.

Theorem 9 Let $M$ be a proper pseudo-slant submanifold of a Cosymplectic manifold $\widetilde{M}$. If $\mathrm{t}$ is parallel, then either $\mathrm{M}$ is a mixed-geodesic or an antiinvariant submanifold. 
Proof. From Theorem 2 and (31) we obtain

$$
\operatorname{nh}(X, Y)=0,
$$

for any $X \in \Gamma\left(D_{\theta}\right)$ and $Y \in \Gamma\left(D^{\perp}\right)$. Also by using (31) and (34), we conclude that

$$
n h(Y, T X)-h\left(Y, T^{2} X\right)=\cos ^{2} \theta h(X, Y)=0 .
$$

This proves our assertion.

Theorem 10 Let $M$ be a proper pseudo-slant submanifold of a cosymplectic manifold $\widetilde{M}$. If $\mathrm{t}$ is parallel, then either $\mathrm{M}$ is a $\mathrm{D}^{\perp}$-geodesic or an antiinvariant submanifold of $\widetilde{M}$.

Proof. If $t$ is parallel, then making use of (32), we obtain

$$
\mathrm{TA}_{\mathrm{NY}} \mathrm{Z}=0 \text {, }
$$

for any $Y, Z \in \Gamma\left(D^{\perp}\right)$. This implies that $M$ is either anti-invariant or $A_{N Y} Z=0$. So we obtain

$$
g(h(Z, W), N Y)=0,
$$

for any $\mathrm{Y}, \mathrm{Z}, \mathrm{W} \in \Gamma\left(\mathrm{D}^{\perp}\right)$. Also by using (32), we conclude that

$$
g\left(A_{n V} Z, Y\right)-g\left(T A_{V} Z, Y\right)=g(h(Y, Z), n V)=0,
$$

for any $V \in \Gamma\left(T^{\perp} M\right)$. This tells us that $M$ is either $D^{\perp}$-geodesic or it is an anti-invariant submanifold.

Given a proper pseudo-slant submanifold $M$ of a Cosymplectic manifold $\widetilde{M}$, if the distributions $D_{\theta}$ and $D^{\perp}$ are totally geodesic in $M$, then $M$ is said to be contact pseudo-slant product.

Theorem 11 Let $M$ be a pseudo-slant submanifold of a cosymplectic manifold $\widetilde{M}$. Then $M$ is a contact pseudo-slant product if and only if the shape operator of $\mathrm{M}$ satisfies

$$
\mathrm{A}_{\mathrm{ND}} \perp \mathrm{TD}_{\theta}=\mathrm{A}_{\mathrm{NTD}_{\theta}} \mathrm{D}^{\perp} .
$$

Proof. Since the ambient space $\widetilde{M}$ is a cosymplectic manifold, for any $X, Y \in$ $\Gamma\left(D_{\theta}\right)$ and $Z \in \Gamma\left(D^{\perp}\right)$, we have

$$
\begin{aligned}
g\left(\nabla_{X} Y, Z\right) & =g\left(\widetilde{\nabla}_{X} \phi Y, \phi Z\right)=g\left(\widetilde{\nabla}_{X} T Y, \phi Z\right)+g\left(\widetilde{\nabla}_{X} N Y, \phi Z\right) \\
& =-g\left(\widetilde{\nabla}_{X} \phi T Y, Z\right)+g\left(\nabla_{X}^{\perp} N Y, N Z\right) \\
& =-g\left(\nabla_{X} T^{2} Y, Z\right)-g\left(\widetilde{\nabla}_{X} N T Y, Z\right)+g\left(\nabla_{X}^{\perp} N Y, N Z\right) \\
& =\cos ^{2} \theta g\left(\nabla_{X} Y, Z\right)+g\left(A_{N T Y} X, Z\right)+g\left(N \nabla_{X} Y, N Z\right)-g(h(X, T Y), N Z),
\end{aligned}
$$


which implies that

$$
\cos ^{2} \theta g\left(\nabla_{X} Y, Z\right)=g\left(A_{N T Y} Z-A_{N Z} T Y, X\right) .
$$

On the other hand, for any $Z, W \in \Gamma\left(D^{\perp}\right)$ and $X \in \Gamma\left(D_{\theta}\right)$, we reach at

$$
\begin{aligned}
g\left(\nabla_{Z} W, X\right)= & -g\left(\widetilde{\nabla}_{Z} X, W\right)=-g\left(\widetilde{\nabla}_{Z} \phi X, \phi W\right) \\
= & -g\left(\widetilde{\nabla}_{Z} T X, \phi W\right)-g\left(\widetilde{\nabla}_{Z} N X, \phi W\right) \\
= & g\left(\widetilde{\nabla}_{Z} \phi T X, W\right)-g\left(\nabla_{Z}^{\perp} N X, N W\right) \\
= & g\left(\nabla_{Z} T^{2} X, W\right)+g\left(\nabla_{Z} N T X, W\right)-g\left(\nabla_{Z}^{\perp} N X, N W\right) \\
= & -\cos ^{2} \theta g\left(\nabla_{Z} X, W\right)-g\left(A_{N T X} Z, W\right)-g\left(N \nabla_{Z} X, N W\right) \\
& -g\left(\left(\nabla_{Z} N\right) X, N W\right) \\
= & \cos ^{2} \theta g\left(\nabla_{Z} W, X\right)-g\left(A_{N T X} Z, W\right)-g\left(N \nabla_{Z} X, N W\right) \\
& +g(h(Z, T X), N W) .
\end{aligned}
$$

This implies that

$$
\cos ^{2} \theta g\left(\nabla_{Z} W, X\right)=g\left(A_{N T X} W-A_{N W} T X, Z\right) .
$$

From (42) and (43), we get desired result.

\section{Pseudo-slant submanifolds in cosymplectic space forms}

In this section, we will study pseudo-slant submanifolds in a cosymplectic space form, give some characterization and submanifold will be characterized.

Theorem 12 Let $M$ be a pseudo-slant submanifold of a cosymplectic space form $\widetilde{M}(\mathrm{c})$ such that $\mathrm{c} \neq 0$. If $\mathrm{M}$ is a curvature-invariant pseudo-slant submanifold, then $M$ is either semi-invariant or anti-invariant submanifold.

Proof. We suppose that $M$ is a curvature-invariant pseudo-slant submanifold of a cosymplectic space form $\widetilde{M}(c)$ such that $c \neq 0$. Then from (29) and (14), we have

$$
g(X, T Z) N Y+g(T Y, Z) N X+2 g(X, T Y) N Z=0,
$$

for any $X, Y, Z \in \Gamma(T M)$. Taking $X=Z$ and $Y=T Z$ in (44), we have

$$
g(T Z, T Z) N Z=0 \text {. }
$$


Here, by using (36) and (37), we obtain

$$
\cos ^{2} \theta \sin ^{2} \theta\left\{g(Z, Z)-\eta^{2}(Z)\right\}^{2}=0 .
$$

This implies that $\sin 2 \theta\left\{g(Z, Z)-\eta^{2}(Z)\right\}=0$, that is, $M$ is either a semiinvariant or an anti-invariant submanifold. Thus the proof is complete.

Theorem 13 Let $M$ be a pseudo-slant submanifold of a cosymplectic space form $\widetilde{M}(\mathrm{c})$ with flat normal connection such that $\mathrm{c} \neq 0$. If $\mathrm{TA}_{\mathrm{V}}=\mathrm{A}_{\mathrm{V}} \mathrm{\top}$ for any vector $\mathrm{V}$ normal to $\mathrm{M}$, then $\mathrm{M}$ is either an anti-invariant or it is a generic submanifold of $\widetilde{M}(\mathrm{c})$.

Proof. If the normal connection of $M$ is flat, then from (26), we have

$$
\begin{aligned}
g\left(\left[A_{\mathrm{U}}, A_{V}\right] X, Y\right)= & \frac{\mathrm{c}}{4}\{\mathrm{~g}(\mathrm{X}, \phi \mathrm{V}) \mathrm{g}(\mathrm{U}, \phi \mathrm{Y})-\mathrm{g}(\mathrm{Y}, \phi \mathrm{V}) \mathrm{g}(\phi X, \mathrm{U}) \\
& +2 \mathrm{~g}(\mathrm{X}, \phi \mathrm{Y}) \mathrm{g}(\phi \mathrm{V}, \mathrm{U})\}
\end{aligned}
$$

for any $X, Y \in \Gamma(T M)$ and $U, V \in \Gamma\left(T^{\perp} M\right)$. Here, choosing $U=n V$ and $\mathrm{Y}=\mathrm{TX}$, by direct calculations, we can state

$$
g\left(\left[A_{V}, A_{n V}\right] X, T X\right)=-\frac{c}{2}\{g(T X, T X) g(n V, n V)\},
$$

that is,

$$
g\left(A_{n V} A_{V} T X-A_{V} A_{n V} T X, X\right)=-\frac{c}{2}\{g(T X, T X) g(n V, n V)\},
$$

from which

$$
\operatorname{tr}\left(A_{n V} A_{V} T\right)-\operatorname{tr}\left(A_{V} A_{n V} T\right)=\frac{c}{2} \operatorname{tr}\left(T^{2}\right) g(n V, n V) .
$$

If $T A_{V}=A_{V} T$, then we conclude that $\operatorname{tr}\left(A_{n V} A_{V} T\right)=\operatorname{tr}\left(A_{V} A_{n V} T\right)$ and thus

$$
\frac{c}{2} \operatorname{tr}\left(T^{2}\right) g(n V, n V)=0,
$$

from here $\operatorname{dim}(T M)=2 q+q+1$, then we can easily to see that

$$
(2 p+q+1) \cos ^{2} \theta g(n V, n V)=0 .
$$

Thus $\theta$ is either $\frac{\pi}{2}$ or $n=0$. This implies that $M$ is either an anti-invariant or it is a generic submanifold. 
Theorem 14 Let $M$ be a proper pseudo-slant submanifold of a cosymplectic space form $\widetilde{M}(\mathrm{c})$. Then the Ricci tensor $\mathrm{S}$ of $\mathrm{M}$ is given by

$$
\begin{aligned}
S(X, W)= & \frac{c}{4}\left\{2 p+q-1+3 \cos ^{2} \theta\right\}(g(X, W)-\eta(X) \eta(W)) \\
& +(2 p+q+1) g(h(X, W), H)-\sum_{l=1}^{2 p+q+1} g\left(h\left(e_{l}, W\right), h\left(X, e_{l}\right)\right)
\end{aligned}
$$

for any $\mathrm{X}, \mathrm{W} \in \Gamma(\mathrm{TM})$.

Proof. For any $\mathrm{X}, \mathrm{Y}, \mathrm{Z} \in \Gamma(\mathrm{TM})$, by using (6) and (12), we have

$$
\begin{aligned}
g(R(X, Y) Z, W)= & \frac{c}{4}\{g(Y, Z) g(X, W)-g(X, Z) g(Y, W) \\
& +\eta(X) \eta(Z) g(Y, W)-\eta(Y) \eta(Z) g(X, W) \\
& +\eta(Y) \eta(W) g(X, Z)-\eta(X) \eta(W) g(Y, Z) \\
& +g(X, \phi Z) g(\phi Y, W)-g(Y, \phi Z) g(\phi X, W) \\
& +2 g(X, \phi Y) g(\phi Z, W)\}+g(h(X, W), h(Y, Z)) \\
& -g(h(Y, W), h(X, Z)) .
\end{aligned}
$$

Now, let $e_{1}, e_{2}, \ldots, e_{p}, e_{p+1}=\sec \theta T e_{1}, e_{p+2}=\sec \theta T e_{2}, \ldots, e_{2 p}=\sec \theta T e_{p}, e_{2 p+1}$ $=\xi, e_{2 p+2}, e_{2 p+3}, \ldots, e_{2 p+q+1}$ be an orthonormal basis of $\Gamma(T M)$ such that $e_{1}, e_{2}, \ldots, e_{p}, e_{p+1}=\sec \theta T e_{1}, e_{p+2}=\sec \theta T e_{2}, \ldots, e_{2 p}=\sec \theta T e_{p}, e_{2 p+1}=$ $\xi$ are tangent to $\Gamma\left(D_{\theta}\right)$ and $e_{2 p+2}, e_{2 p+3}, \ldots, e_{2 p+q+1}$ are tangent to $\Gamma\left(D^{\perp}\right)$. Hence, from (46) taking $Y=Z=e_{i}, e_{j}, e_{k}$ and $1 \leq i \leq p, 1 \leq j \leq p, \xi, 2 p+2 \leq$ $k \leq 2 p+q+1$ then, we obtain

$$
\begin{aligned}
S(X, W)= & \sum_{i=1}^{p} g\left(R\left(X, e_{i}\right) e_{i}, W\right)+\sum_{j=p+1}^{2 p} g\left(R\left(X, \sec \theta T e_{j}\right) \sec \theta T e_{j}, W\right) \\
& +g(R(X, \xi) \xi, W)+\sum_{k=2 p+2}^{2 p+q+1} g\left(R\left(X, e_{k}\right) e_{k}, W\right) \\
= & \frac{c}{4}\{(2 p+q) g(X, W)\}-\frac{c}{4}\{(2 p+q-1) \eta(X) \eta(W) \\
& \left.+3 \cos ^{2} \theta[g(X, W)-\eta(X) \eta(W)]-g(X, W)\right\} \\
& +(2 p+q+1) g(h(X, W), H)-\sum_{i=1}^{p} g\left(h\left(e_{i}, W\right), h\left(X, e_{i}\right)\right)
\end{aligned}
$$




$$
\begin{aligned}
& -\sum_{j=p+1}^{2 p} g\left(h\left(\sec \theta T e_{j}, W\right), h\left(X, \sec \theta T e_{j}\right)\right)+g(h(\xi, W), h(X, \xi)) \\
& -\sum_{k=2 p+2}^{2 p+q+1} g\left(h\left(e_{k}, W\right), h\left(X, e_{k}\right)\right) .
\end{aligned}
$$

Here

$$
\begin{gathered}
\sum_{l=1}^{2 p+q+1} g\left(h\left(e_{l}, W\right), h\left(X, e_{l}\right)=\sum_{i=1}^{p} g\left(h\left(e_{i}, W\right), h\left(X, e_{i}\right)\right.\right. \\
+\sum_{j=p+1}^{2 p} g\left(h\left(\sec \theta T e_{j}, W\right), h\left(X, \sec \theta T e_{j}\right)\right) \\
+\sum_{k=2 p+2}^{2 p+q+1} g\left(h\left(e_{k}, W\right), h\left(X, e_{k}\right)\right)
\end{gathered}
$$

hance, we have

$$
\begin{aligned}
S(X, W)= & \frac{c}{4}\left\{2 p+q-1+3 \cos ^{2} \theta\right\}(g(X, W)-\eta(X) \eta(W)) \\
& +(2 p+q+1) g(h(X, W), H)-\sum_{l=1}^{2 p+q+1} g\left(h\left(e_{l}, W\right), h\left(X, e_{l}\right)\right)
\end{aligned}
$$

the proof is complete.

Theorem 15 Let $M$ be a pseudo-slant submanifold of a cosymplectic space form $\widetilde{M}(\mathrm{c})$. Then the scalar curvature $\rho$ of $\mathrm{M}$ is given by

$$
\left.\rho=\frac{c}{4}\left\{2 p+q-1+3 \cos ^{2} \theta\right\}(2 p+q)+(2 p+q+1)^{2} \| H\right)\left\|^{2}-\right\| h \|^{2} .
$$

Proof. By using (45), we have

$$
\rho=\sum_{l=1}^{2 p+q+1} S\left(e_{l}, e_{l}\right)
$$

which gives (47). Thus the proof is complete. 
Theorem 16 Let $M$ be a proper pseudo-slant submanifold of a cosymplectic space form $\widetilde{M}(\mathrm{c})$ such that $\mathrm{c} \neq 0$. Every totally umbilical pseudo-slant submanifold $M$ in a cosymplectic space form $\widetilde{M}(c)$ is a semi-invariant or antiinvariant submanifold.

Proof. We suppose that $M$ is totally umbilical pseudo-slant submanifold in cosymplectic space form $\widetilde{M}(c)$. Since $M$ is totally geodesic, we have

$$
g(\widetilde{R}(X, Y) Z, \phi Z)=g\left(\left(\nabla_{X} h\right)(Y, Z)-\left(\nabla_{Y} h\right)(X, Z), \phi Z\right)=0,
$$

or

$$
\begin{aligned}
g(\widetilde{R}(X, Y) Z, \phi Z)= & g\left(\nabla_{X}^{\perp} g(Y, Z) H-g\left(\nabla_{X} Y, Z\right) H-g\left(\nabla_{X} Z, Y\right) H, \phi Z\right) \\
& -g\left(\nabla_{Y}^{\perp} g(X, Z) H-g\left(\nabla_{Y} X, Z\right) H-g\left(\nabla_{Y} Z, X\right) H, \phi Z\right)=0
\end{aligned}
$$

for any $X, Y \in \Gamma\left(D_{\theta}\right)$ and $Z \in \Gamma\left(D^{\perp}\right)$. Since the ambient space $M$ is a cosymplectic space form, from (6) we infer

$$
g(\widetilde{R}(X, Y) Z, \phi Z)=\frac{c}{2} g(X, \phi Y) g(N Z, N Z)=0 .
$$

Taking $\mathrm{Y}=\mathrm{TX}$ in equation (48), we have

$$
g(X, \phi T X) g(N Z, N Z)=0 .
$$

Here, by using (36) and (37), we obtain

$$
\cos ^{2} \theta \sin ^{2} \theta g(Z, Z)\left\{g(X, X)-\eta^{2}(X)\right\}=0 .
$$

This implies that $\sin 2 \theta=0$, that is, $M$ is either a semi-invariant or an antiinvariant submanifold. This proves our assertion.

Theorem 17 Let $M$ be a totally umbilical pseudo-slant submanifold of a Cosymplectic space form $\widetilde{M}(\mathrm{c})$. Then the Ricci tensor $\mathrm{S}$ of $\mathrm{M}$ is given by

$$
S(X, W)=\frac{c}{4}\left\{2 p+q-1+3 \cos ^{2} \theta\right\}(g(X, W)-\eta(X) \eta(W))
$$

for any $\mathrm{X}, \mathrm{W} \in \Gamma(\mathrm{TM})$.

Proof. From by using (11) and (45), we obtain

$$
\begin{aligned}
S(X, W)= & \frac{c}{4}\left\{2 p+q-1+3 \cos ^{2} \theta\right\}(g(X, W)-\eta(X) \eta(W)) \\
& +(2 p+q+1) g(g(X, W) H, H)-\sum_{l=1}^{2 p+q+1} g\left(g\left(e_{l}, W\right) H, g\left(X, e_{l}\right) H\right)
\end{aligned}
$$

this complete the proof. Thus we have the following corollary. 
Corollary 2 Every totally umbilical pseudo-slant submanifold $M$ of a cosymplectic space form $\widetilde{M}(\mathrm{c})$ is an $\eta$-Einstein submanifold.

Theorem 18 Let $M$ be a totally umbilical pseudo-slant submanifold of a cosymplectic space form $\widetilde{M}(\mathrm{c})$. Then the scalar curvature $\rho$ of $\mathrm{M}$ is given by

$$
\rho=\frac{c}{4}\left\{2 p+q-1+3 \cos ^{2} \theta\right\}(2 p+q) .
$$

Proof. By using (49), we have

$$
\rho=\sum_{l=1}^{2 p+q+1} S\left(e_{l}, e_{l}\right)
$$

which gives (50). Thus the proof is complete.

Example 1 Let $M$ be a submanifold of $\mathbb{R}^{9}$ defined by $\chi(u, v, s, w, z)=(u,-\sqrt{2} v, v \sin \alpha, v \cos \alpha, s \cos w,-\cos w, s \sin w,-\sin w, z)$.

We can easily to see that the tangent bundle of $\mathrm{M}$ is spanned by the tangent vectors

$$
\begin{aligned}
& e_{1}=\frac{\partial}{\partial x_{1}}, e_{5}=\xi=\frac{\partial}{\partial z}, \\
& e_{2}=-\sqrt{2} \frac{\partial}{\partial y_{1}}+\sin \alpha \frac{\partial}{\partial x_{2}}+\cos \alpha \frac{\partial}{\partial y_{2}}, \\
& e_{3}=\cos w \frac{\partial}{\partial x_{3}}+\sin w \frac{\partial}{\partial x_{4}}, \\
& e_{4}=-\sin w \frac{\partial}{\partial x_{3}}+\sin w \frac{\partial}{\partial y_{3}}+s \cos w \frac{\partial}{\partial x_{4}}-\cos w \frac{\partial}{\partial y_{4}} .
\end{aligned}
$$

We define the almost contact structure $\phi$ of $\mathbb{R}^{9}$, by

$$
\phi\left(\frac{\partial}{\partial x_{i}}\right)=\frac{\partial}{\partial y_{i}}, \quad \phi\left(\frac{\partial}{\partial y_{j}}\right)=-\frac{\partial}{\partial x_{j}}, \quad \phi\left(\frac{\partial}{\partial z}\right)=0, \quad 1 \leq i, j \leq 4 .
$$

For any vector field $\mathrm{X}=\lambda_{i} \frac{\partial}{\partial x_{i}}+\mu_{j} \frac{\partial}{\partial y_{i}}+\nu \frac{\partial}{\partial z} \in \Gamma\left(T \mathbb{R}^{9}\right)$, then we have

$$
g(X, X)=\lambda_{i}^{2}+\mu_{j}^{2}+v^{2}, \quad g(\phi X, \phi X)=\lambda_{i}^{2}+\mu_{j}^{2}
$$


and

$$
\phi^{2} X=-\lambda_{i} \frac{\partial}{\partial x_{i}}-\mu_{j} \frac{\partial}{\partial y_{i}}=-X+\eta(X) \xi
$$

for any $i, j=1,2,3,4$. It follows that $g(\phi X, \phi X)=g(X, X)-\eta^{2}(X)$. Thus $(\phi, \eta, \xi, g)$ is an almost contact metric structure on $\mathbb{R}^{9}$. Thus we have

$$
\begin{gathered}
\phi e_{1}=\frac{\partial}{\partial y_{1}}, \\
\phi e_{2}=\sqrt{2} \frac{\partial}{\partial x_{1}}+\sin \alpha \frac{\partial}{\partial y_{2}}-\cos \alpha \frac{\partial}{\partial x_{2}}, \\
\phi e_{3}=\cos w \frac{\partial}{\partial y_{3}}+\sin w \frac{\partial}{\partial y_{4}}, \\
\phi e_{4}=-\sin w \frac{\partial}{\partial y_{3}}-\sin w \frac{\partial}{\partial x_{3}}+s \cos w \frac{\partial}{\partial y_{4}}+\cos w \frac{\partial}{\partial x_{4}} .
\end{gathered}
$$

By direct calculations, we can infer $\mathrm{D}_{\theta}=\operatorname{span}\left\{\mathrm{e}_{1}, \mathrm{e}_{2}\right\}$ is a slant distribution with slant angle $\cos \theta=\frac{g\left(e_{1}, \phi e_{2}\right)}{\left\|e_{1}\right\|\left\|\phi e_{2}\right\|}=\frac{\sqrt{6}}{3}, \theta=\cos ^{-1}\left(\frac{\sqrt{6}}{3}\right)$. Since $g\left(\phi e_{3}, e_{i}\right)=0$, $i=1,2,4,5$ and $g\left(\phi e_{4}, e_{j}\right)=0, j=1,2,3,5, \phi e_{3}$, $\phi e_{4}$ are orthogonal to $\mathrm{M}, \mathrm{D}^{\perp}=\operatorname{span}\left\{e_{3}, e_{4}\right\}$ is an anti-invariant distribution. Thus $\mathrm{M}$ is a 5 dimensional proper pseudo-slant submanifold of $\mathbb{R}^{9}$ with its usual almost contact metric structure.

Let $\nabla$ be the Levi-Civita connection on $\mathbb{R}^{9}$. Then we have

$$
\begin{aligned}
0= & {\left[e_{1}, e_{1}\right]=\left[e_{2}, e_{2}\right]=\left[e_{3}, e_{3}\right]=\left[e_{4}, e_{4}\right]=\left[e_{5}, e_{5}\right] } \\
= & {\left[e_{1}, e_{2}\right]=\left[e_{1}, e_{3}\right]=\left[e_{1}, e_{4}\right]=\left[e_{1}, e_{5}\right]=\left[e_{2}, e_{3}\right] } \\
= & {\left[e_{2}, e_{4}\right]=\left[e_{2}, e_{5}\right]=\left[e_{3}, e_{5}\right]=\left[e_{4}, e_{5}\right] } \\
{\left[e_{3}, e_{4}\right]=} & \left(\frac{\cos 2 w}{\sin w}\right) \frac{\partial}{\partial x_{3}}+\left(\frac{1}{\sin w}\right) \frac{\partial}{\partial y_{3}}-\left(\frac{1}{\cos w}\right) \frac{\partial}{\partial x_{4}} \\
& +\left(\frac{(s-1)}{s} \cos w\right) \frac{\partial}{\partial y_{4}},
\end{aligned}
$$

and

$$
g\left(e_{1}, e_{1}\right)=g\left(e_{3}, e_{3}\right)=1, g\left(e_{2}, e_{2}\right)=3, g\left(e_{4}, e_{4}\right)=s^{2}+1, g\left(e_{5}, e_{5}\right)=1,
$$




$$
\begin{aligned}
& g\left(e_{1}, e_{2}\right)=g\left(e_{1}, e_{3}\right)=g\left(e_{1}, e_{4}\right)=g\left(e_{1}, e_{5}\right)=0, \\
& g\left(e_{2}, e_{3}\right)=g\left(e_{2}, e_{4}\right)=g\left(e_{2}, e_{5}\right)=0, \\
& g\left(e_{3}, e_{4}\right)=g\left(e_{3}, e_{5}\right)=g\left(e_{4}, e_{5}\right)=0 .
\end{aligned}
$$

Using Koszul's formula, the Riemannian connection $\nabla$ of the metric $\mathrm{g}$ is given by

$$
\begin{aligned}
2 g\left(\nabla_{X} Y, Z\right)= & X g(Y, Z)+Y g(Z, X)-Z g(X, Z) \\
& -g(X,[Y, Z])-g(Y,[X, Z])-g(Z,[X, Y])
\end{aligned}
$$

Koszul's formula yields, we can find

$$
\begin{aligned}
& 0=\nabla_{e_{1}} e_{1}=\nabla_{e_{1}} e_{2}=\nabla_{e_{1}} e_{3}=\nabla_{e_{1}} e_{4}=\nabla_{e_{1}} e_{5} \\
& =\nabla_{e_{2}} e_{2}=\nabla_{e_{2}} e_{4}=\nabla_{e_{2}} e_{5}=\nabla_{e_{3}} e_{1}=\nabla_{e_{3}} e_{2} \\
& =\nabla_{e_{3}} e_{5}=\nabla_{e_{4}} e_{5}=\nabla_{e_{5}} e_{5}, \quad \nabla_{e_{4}} e_{4}=-s e_{3}, \quad \nabla_{e_{3}} e_{3}=\frac{1}{s} e_{3} \text {, } \\
& \nabla_{e_{3}} e_{4}=\frac{s}{s^{2}+1}\left(1-s^{2}+\left(1-s-s^{2}\right) \cos ^{2} w+s^{2} \sin ^{2} w\right) e_{4} \\
& +\left(\frac{\cos 2 w-\tan ^{2} w}{\tan w}\right) e_{3} \text {. }
\end{aligned}
$$

Thus we can say that $\mathrm{M}$ is $\mathrm{D}_{\theta}$-geodesic and mixed-geodesic. But it is not $\mathrm{D}^{\perp}$ geodesic.

\section{References}

[1] A. Carriazo, New developments in slant submanifolds theory, Narasa Publishing Hause New Delhi, India, 2002.

[2] A. Lotta, Slant submanifolds in contact geometry, Bulletin Mathematical Society Roumanie, 39 (1996), 183-198.

[3] B. Y. Chen, Geometry of slant submanifolds, Katholieke Universiteit Leuven, Leuven, Belgium, View at Zentralblatt Math., 1990.

[4] B. Y. Chen, Slant immersions, Bull. Austral. Math. Soc., 41 (1990), 135147. 
[5] S. Dirik, M. Atçeken, Pseudo-slant submanifolds of nearly Cosymplectic manifold, Turkish Journal of Mathematics and Computer Science, article ID 20140035, 14 pages, (2013).

[6] D. Blair, Contact manifolds in Riemannian geometry, Lecture Notes in Mathematic Springer-Verlag, New York, Vol. 509 (1976).

[7] J. L. Cabrerizo, A. Carriazo, L. M. Fernandez, M. Fernandez, Slant submanifolds in Sasakian manifolds, Glasgow Math. J., 42 (2000), 125-138.

[8] M. Atçeken, S. K. Hui, Slant and pseudoslant submanifolds in (LCS $)_{n^{-}}$ manifolds, Czechoslovak Math. J., 63 (2013), 177-190.

[9] M. Atçeken, S. Dirik, On the geometry of pseudo-slant submanifolds of a Kenmotsu manifold, Gulf Joural of Mathematics, 2 (2014), 51-66.

[10] M. Atçeken, S. Dirik, On contact CR-submanifolds of Kenmotsu manifolds, Acta Univ. Sapientiae, Mathematica, 4 (2012), 182-198.

[11] M. A. Khan, S. Uddin, K. Singh, A classification on totally umbilical proper slant and hemi-slant submanifolds of a nearly trans-Sasakian manifold, Differential Geometry - Dynamical Systems, 13 (2011), 117-127.

[12] N. Papaghuic, Semi-slant submanifolds of a Kaehlarian manifold, An. St. Univ. Al. I. Cuza. Univ. Iasi, 40 (2009), 55-61.

[13] S. Uddin, C. Ozel, V. A. Khan, A classification of a totally umbilical slant submanifold of Cosymplectic manifolds, Hindawi puplishing corporation abstract applied analysis, article ID 716967, 8 pages (2012).

[14] U. C. De, A. Sarkar, On pseudo-slant submanifolds of trans Sasakian manifold, Proceedings of the Estonian Academy of sciences, 60 (1) (2011), $1-11$.

[15] V. A. Khan, M. A. Khan, Pseudo-slant submanifolds of a Sasakian manifold, Indian J. Pure Appl. Math., 38 (2007), 31-42. 